\title{
Artículos
}

\section{Generación de residuos orgánicos en las unidades económicas comerciales y de servicios en la Ciudad de México}

\section{Organic waste production in commercial and services economic units in Mexico City}

\author{
Cecilia Esther Muñoz Cadena* \\ Rosalba Esther Morales Pérez**
}

\section{Resumen}

La inconsistencia en los datos oficiales respecto a los inventarios de residuos afecta a diversos países del orbe. Dada su importancia en la planeación de la gestión integral para mitigar sus impactos, este artículo tiene por objeto estimar la fracción orgánica de los residuos de manejo especial generados en las unidades económicas comerciales y de servicios de la Ciudad de México, mediante el análisis de las tasas de generación por empleado. Los resultados arrojan una generación de 14155 toneladas diarias de residuos orgánicos. Esta estrategia alternativa permitirá reforzar la gestión integral y la implementación de la política de residuos.

Palabras clave: residuos orgánicos; unidades económicas comerciales y de servicios; Ciudad de México; generación de residuos.

\begin{abstract}
The inconsistency of official data on waste inventories affects several countries in the world. Given their importance in integral management planning to mitigate its impact, this article seeks to estimate the organic share of special management waste generated in the commercial and service economic units of Mexico City, by analyzing

* Huerto Urbano de los Niños y las Niñas. Dirección postal: Av. Insurgentes Norte 694, col. Santa María Insurgentes, delegación Cuauhtémoc, Ciudad de México, México. Correo electrónico: ceci_esther@yahoo.com.mx; cmunozc0901@egresado.ipn.mx.

** Centro Interdisciplinario de Investigaciones y Estudios sobre Medio Ambiente y Desarrollo-IPN. Dirección postal: Calle 30 de Junio de 1520 s/n, Barrio la Laguna Ticomán, 07340, Gustavo A. Madero, Ciudad de México, México. Correo electrónico: rosalbaesther@, yahoo.com.mx
\end{abstract}


generation rates per employee. The results show the daily generation of 14155 tons of organic waste. This alternative strategy will reinforce integral management and the implementation of waste policy.

Keywords: organic waste; commerce and services economic units; Mexico City; waste generation.

\section{Introducción}

Existe una relación directa entre las actividades e insumos requeridos por los centros urbanos y los residuos que éstos generan (Rodríguez, 2004, p. 1), por lo que, para lograr una correcta gestión integral es indispensable contar con inventarios confiables y actualizados sobre la generación y composición de los residuos sólidos. Así, dichos inventarios constituyen una poderosa herramienta de planeación para el diseño de estrategias adecuadas en el manejo integral de los residuos. Sin embargo, en la mayor parte de los países en vías de desarrollo los datos estadísticos sobre la generación de residuos sólidos urbanos (RSU) son inconsistentes, ya que provienen de fuentes diversas (Miezah et al., 2015, p. 15; Wilson et al., 2015, p. 329).

La Ciudad de México (CDMX) no es la excepción. En ella, la política de residuos se define como el conjunto de acciones, operaciones y procesos que permiten disminuir la cantidad de residuos existentes en cada una de sus etapas del manejo: generación, almacenamiento in situ, recolección, tratamiento y disposición adecuada para su prevención y minimización; siendo el Programa de Gestión Integral de los Residuos Sólidos para la Ciudad de México el instrumento rector (PGIRS, 2016, pp. 5-6).

El artículo $6^{\circ}$ de la Ley de Residuos Sólidos del DF (LRSDF, 2003) establece que la Secretaría del Medio Ambiente (Sedema) debe integrar un inventario de residuos sólidos y sus fuentes generadoras, en coordinación con la Secretaría de Obras y Servicios Públicos (Sobse) y las delegaciones políticas (fracc. V); así como integrar un registro actualizado de los planes de manejo (fracc. X11l). Si bien la Sedema ha cumplido con la difusión anual del inventario, al año 2013 carecía de un sistema de información que integrara los datos históricos de generación de RSU, su caracterización física y su tendencia.

A partir de 2011, los inventarios de residuos anuales estimaron el porcentaje del aporte de los principales sectores generadores: domiciliario (47.7\%), comercial (15.4\%), servicios (13.6\%), mercados (10.6\%), Central de Abasto (4.6\%), diversos (5.1\%) y controlados (3\%). Los dos últimos se refieren a los residuos de reclusorios, hospitales y de manejo especial (Se- 
dema, 2014, p. 13; Sedema, 2015, p. 13). Así, los residuos de los sectores comerciales, de servicios, de mercados, incluyendo la Central de Abasto, representaban en conjunto el $44.2 \%$ de la generación de residuos sólidos de la ciudad.

Estos sectores constituían el $18.4 \%$ de las actividades económicas del producto interno bruto nacional (PIB). En este sentido, Sobrino (2016, p. 18) apunta a que los censos económicos pueden aportar datos sobre las principales características de las unidades económicas a escala municipal, por lo que es posible referenciar sus cifras de crecimiento a los datos de los últimos censos económicos $(2009 ; 2014)$, donde se observó un aumento del 4\% en el número de unidades económicas dedicadas al comercio. En la misma proporción creció el número de personal ocupado, con una producción bruta total de $10 \%$, lo que reflejaba el crecimiento del sector económico y la estructura de las actividades realizadas (INEGI, 2014). Sin embargo, para el segundo semestre del 2016, el Directorio Estadístico Nacional de Unidades Económicas (DENUE) reportó un aumento en este tipo de unidades de 50.62\% respecto al reporte del 2014 (INEGI, 2016a), mientras que la generación de RSU, para el mismo periodo, aumentó menos del 1\% (Sedema, 2015, pp. 11, 14). En el Cuadro 1 se muestran estas tendencias.

Cabe señalar en este punto que existen estudios sobre la generación de RSU en la CDMX donde se han reportado datos que rebasan las cifras oficiales publicadas por la Sedema (CIIEMAD- IPN, 2013; Wismer y López de Alba, 2011, p. 4; JICA-GDF, 1999, p. 44).

\section{Cuadro 1}

Comportamiento de las Unidades Económicas (UE) en la Ciudad de México y sus RSU generados. Periodos: 2004-2016

\begin{tabular}{ccc}
\hline Periodo & $\boldsymbol{U} \boldsymbol{R}$ & $\boldsymbol{R S U}(\boldsymbol{t} / \boldsymbol{a n ̃ o})$ \\
\hline $2004-2005$ & 223311 & 4459570 \\
$2009-2010$ & 247346 & 4567245 \\
$2013-2014$ & 229414 & 4677840 \\
$2015-2016$ & 464583 & 4705945 \\
\hline
\end{tabular}

Fuente: Censos Económicos (2009; 2014), DENUE (2016a), Sedema (2009; 2012; 2013; 2014; 2015), PGIRS (2004; 2010; 2016). 


\subsection{Clasificación y manejo de los residuos sólidos en la Ciudad de México}

La Ley General para la Prevención y Gestión Integral de Residuos (DOF, 2003) establece la clasificación de los residuos sólidos con base en sus características y origen. Los RSU son generados en casas habitación y provienen de materiales eliminados de las actividades domésticas, así como de establecimientos o vías públicas con características domiciliarias. Por otra parte, los residuos peligrosos son aquellos que presentan características de corrosividad, reactividad, explosividad, toxicidad, inflamabilidad o que portan agentes infecciosos.

Finalmente, los residuos de manejo especial (RME) son los generados en los procesos industriales y por las actividades comerciales y de servicios, como oficinas, comedores, sanitarios y mantenimiento. Por sus características se consideran como RSU; sin embargo, por su volumen de generación, superior a 10 t/año, se rigen por la Norma Oficial Mexicana NOM-161-Semarnat-2011 (DOF, 2013a, p. 14).

Como lo menciona el Diagnóstico Básico para la Gestión Integral de Residuos (INECC, 2012, p. 60), las fuentes generadoras de los RME, que incluyen actividades de los sectores primario a terciario, se encuentran registradas en el Directorio Estadístico Nacional de Unidades Económicas (DENUE) del INEGI. Este diagnóstico menciona que es posible distinguir a los grandes generadores de residuos de los pequeños. En los primeros, el control lo realizan las autoridades ambientales de las entidades federativas, mientras que los segundos son las mismas fuentes generadoras quienes deben ocuparse de su propio manejo a través de empresas especializadas. Es bajo esta última clasificación que son considerados los residuos de este trabajo, ya que son RSU de grandes volúmenes, resultado del consumo y producción de procesos comerciales y de servicios.

Los RSU se dividen en: $i$ ) residuos orgánicos (RO): alimentos y poda, y ii) residuos inorgánicos (RI): vidrio, papel, cartón, plástico, laminados de materiales reciclables, aluminio y metales no peligrosos (LRSDF, 2003: art.19; Reglamento de la LRSDF, 2008: art. 13-14; Sedema, 2013a).

La Figura 1 muestra la clasificación de los residuos sólidos en la CDMX tanto por su composición como por sus fuentes generadoras; se hace énfasis en la relación que guardan las unidades económicas comerciales y de servicios (UECS) con potencial para generar estos RO de manejo especial.

Con base en la fuente de generación, la Dirección General de Servicios Urbanos adscrita a la Sobse clasificó los RSU en: domiciliarios, comerciales (establecimientos y mercados), servicios (restaurantes, bares, centros 


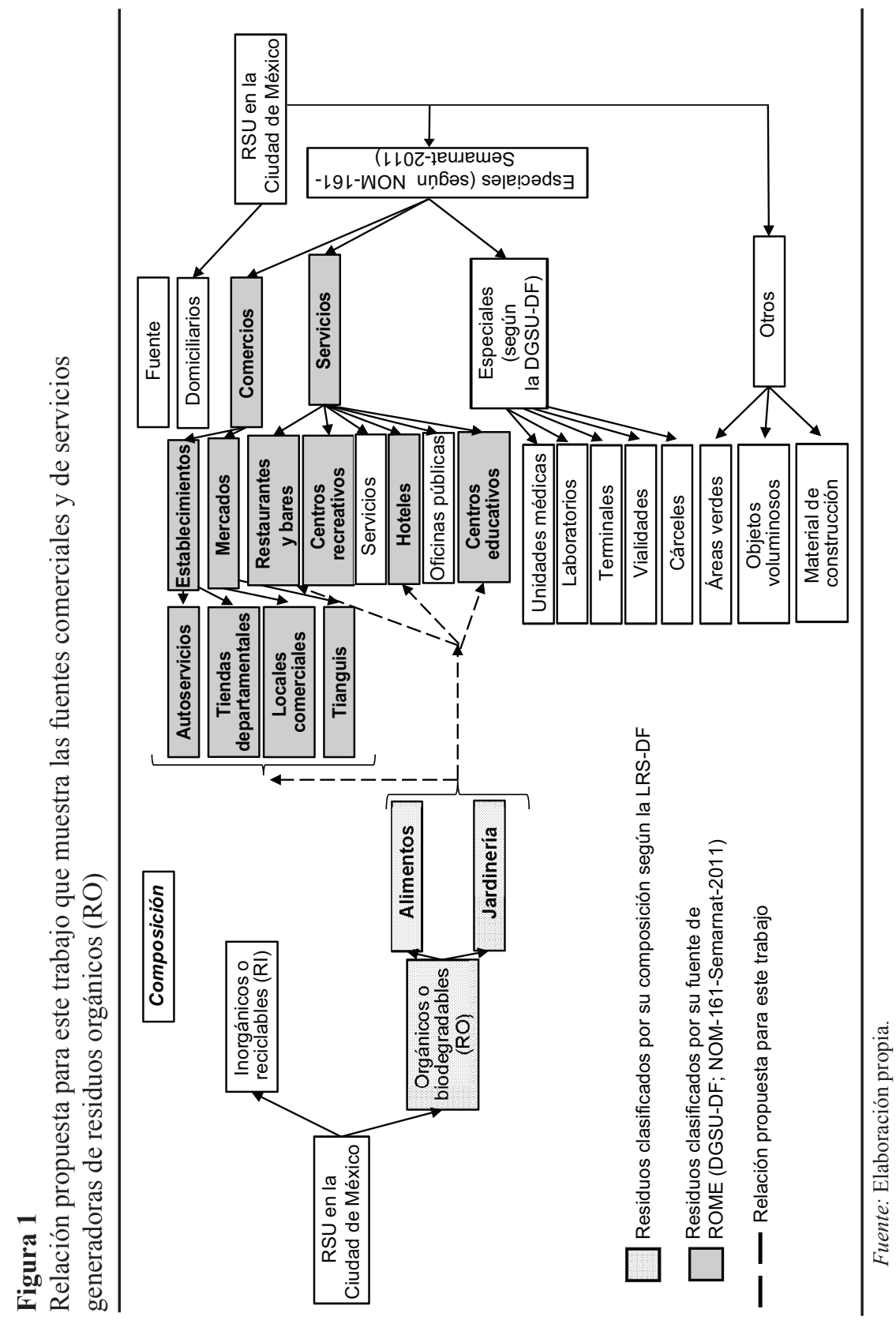


recreativos, servicios públicos, hoteles, oficinas públicas y centros educativos), especiales (unidades médicas y laboratorios, terminales, vialidades, cárceles) y otros (áreas verdes, objetos voluminosos y materiales de construcción) (JICA-GDF, 1999, p. 9).

De acuerdo con la LRSDF (2003: art. 22) y su norma ambiental NADF024-AMBT-2013 (Sedema, 2013a), la recolección segregada de los RSU en las fracciones orgánica e inorgánica en las vialidades primarias está a cargo de la Sobse. Mientras que en las vialidades secundarias esa atribución corresponde a las 16 delegaciones políticas de la CDMX. Así, éstas transportan los RSU a las estaciones de transferencia por conducto de la Agencia de Gestión Urbana, adscrita a la Sobse (AGU, 2017), donde se realiza el trasvase a vehículos de gran tonelaje, que son enviados a instalaciones de tratamiento o a sitios de disposición final.

De acuerdo con la Sedema (2014, p. 32), durante el año 2013 se enviaron $1942 \mathrm{t} / \mathrm{d}$ de RO a las plantas de composteo con las que cuenta la ciudad. Al siguiente año, la recolección de orgánicos para composteo se redujo a 1690 t/d (Sedema; 2015, p. 25). Un punto importante a destacar es la eficiencia reportada en la recolección selectiva de los residuos orgánicos (RO) por las delegaciones encargadas de la recolección en las fuentes domiciliarias, comerciales y de servicios. Para 2013, la eficiencia promedio de recolección fue de $58 \%$ para este tipo de residuos (Sedema, 2014, p. 8) y al año siguiente disminuyó a 48\% (Sedema, 2015, p. 14).

\subsection{Tendencias y métodos de estimación de la generación de los RSU en la Ciudad de México}

Según el Programa de las Naciones Unidas para los Asentamientos Humanos (ONU-Habitat, 2012, p. VII), el crecimiento demográfico de las ciudades de América Latina y el Caribe disminuyó a partir del año 2000, siendo menor al 2\% entre 1995 y 2012. En tanto, el aumento en la generación de RSU se elevó $65 \%$. Esto proviene de varios factores: el cambio en las actividades económicas, el mayor poder adquisitivo de la población y los patrones de consumo en contraposición a la incipiente valorización y reciclaje de residuos. En este contexto, la planificación urbana constituye el punto de partida hacia la sostenibilidad económica, social y medioambiental.

La población de la CDMX ha seguido esta misma tendencia, aunque en menor grado, de acuerdo con las cifras oficiales. El aumento poblacional durante el periodo 2005-2015 fue del 2.3\% (INEGI, 2016, p. 3.1), mientras que la generación de RSU entre 2004 y 2015 se elevó 7.4\%, al pasar de 
12000 t/d (1 394 kg/d per cápita) a 12893 t/d (1.46 kg/d per cápita) (Gobierno del Distrito Federal, 2004, p. 23; Sedema, 2015, p. 11).

De acuerdo con los Inventarios de Residuos Sólidos de 2011 a 2014, la generación de RSU por sector o fuentes de generación reportó los siguientes datos: en viviendas aumentó 147 t/d; en comercios y servicios aumentó 53 t/d; en la Central de Abasto no registró cambio, y en los mercados (fijos y ambulantes) disminuyó 43 t/d (Sedema, 2012, p. 9; Sedema, 2013, p. 21; Sedema, 2014, p. 4; Sedema, 2015, p. 12).

No obstante dicha disminución, se han realizado estudios especializados en los mercados públicos (fijos) en la Ciudad de México que reportan una generación de $732.11 \mathrm{t} / \mathrm{d}$ en 10 delegaciones. Esto corrobora la carencia de datos para este sector en los inventarios de residuos sólidos, puesto que los mercados fijos no cuentan con planes de manejo; por ello resulta difícil estimar el aporte de la generación en el sector de los mercados (Morales, 2016, pp. 139, 151). El mismo caso aplica para la Central de Abasto (Morales, 2011, p. 9).

Los datos sobre la composición física de los RSU generados en la CDMX se reportan dentro de los PGIRS. Con base en todas las fuentes generadoras, el primero, 2004-2010, calculó 43\% de materia orgánica, $40 \%$ de material reciclable y $17 \%$ de otros materiales, así como la producción de 10 t/d (Gobierno del Distrito Federal, 2004, pp. 25-26).

El segundo PGIRS, 2010-2015, arrojó que 55.58\% de la generación de RSU, correspondía a los RO; $20.30 \%$ a los RI con potencial reciclable, y $24.12 \%$ tenía escaso valor en el mercado (Gobierno del Distrito Federal, 2010 , p. 17). De esta manera, la generación de los RO aumentó $12.58 \%$ entre 2004 y 2010, según las cifras oficiales. El tercer PGIRS, 2016-2020, reportó $44.06 \%$ de generación estimada para los RO, para los RI con potencial reciclable de $18.3 \%$ en el mercado para los RI de aprovechamiento limitado, $0.21 \%$ para los RME o voluminoso, y $0 \%$ para los residuos provenientes de fuentes distintas a los establecimientos comerciales, industriales y de servicios; sin embargo, según la fuente, estos porcentajes se obtuvieron en el 2009 (Sedema, 2015, p. 10).

Para estimar la generación y composición de RSU existen dos métodos básicos: el muestreo y el flujo de materiales (Tchobanoglous y Kreith, 2002, capítulo 5.2). El método más utilizado por las instancias locales está basado en el propuesto por la Organización Panamericana y Mundial de la Salud (Jiménez, 2001, pp. 457-458), realizado a través del análisis sectorial de residuos sólidos en México, elaborado tanto por la Asociación Mexicana para el Control de Residuos Sólidos Peligrosos, como por la Dirección General de Residuos Sólidos, Materiales y Riesgo del Instituto Nacional de Ecología- 
INE/DGMR (OPS-OMS, 1996). En este caso, la generación de RSU fue estimada por muestreos periódicos, donde se determinaban tanto los parámetros de generación por fuente, como su composición física y química.

Posteriormente, en el primer PGIRS, la Sobse, por conducto de la Dirección General de Servicios Urbanos, reportó que utilizaba las estadísticas de ingresos a las estaciones de transferencia, plantas de selección y sitios de disposición final, donde se determinaban los volúmenes promedio por delegación (PGIRS, 2004, p. 23). Sin embargo, en los subsecuentes PGIRS no se describieron claramente los métodos de estimación para la generación de residuos en la Ciudad de México.

Por su parte, los métodos para estimar la generación de residuos unitarios utilizados para el estudio realizado por la Agencia de Cooperación Internacional Japonesa y el Gobierno del Distrito Federal, se hicieron mediante investigaciones de campo en sectores y subsectores económicos a través de la DGSU. Así, las proporciones obtenidas fueron utilizadas a lo largo de todo el documento generado (JICA/GDF, 1999, p. 11).

Las cifras sobre la generación de RSU, tanto a nivel nacional como local, siguen presentando limitaciones importantes, ya que no se realizan mediciones directas. Estas cifras son calculadas por la Secretaría de Desarrollo Social, o bien la DGSU en el orden local, donde se realiza anualmente una estimación de la generación de residuos en cada delegación, utilizando índices para la generación de residuos en domicilios, establecimientos mercantiles y de servicios, así como otras fuentes, calculados con base en estudios realizados con anterioridad (PGIRS, 2016, pp. 5-6). Estos mismos índices se calculan conforme a lo establecido en la norma NMX-AA-61-1985 sobre la Determinación de la Generación de Residuos Sólidos, la cual establece un procedimiento a partir de la información obtenida de un muestreo estadístico aleatorio de campo, con duración de ocho días para cada estrato socioeconómico, mediante el cual se pesan los residuos generados (Secofi, 1985, capítulo. 6.1; Semarnat, 2012, p. 434).

Otra forma de estimar la generación de residuos en diversas ciudades de México es la presentada por el Instituto Nacional de Ecología, en la que se hizo una proyección de 15 años hasta el nivel de localidad, mediante un modelo de extrapolación hiperbólica, donde se utilizaron datos poblacionales de los Censos Nacionales de Población y Vivienda y su tasa esperada de crecimiento, provista por el Consejo Nacional de Población, para obtener las cifras de crecimiento poblacional promedio. Para cada localidad se identificaron las principales actividades económicas que fueron clasificadas en tres grandes grupos: 1) agrícola e industrial, 2) comercial y servicios, y 3) turísticos. Los resultados fueron analizados junto con la generación per cápita de 
residuos sólidos municipales por localidad. Así se obtuvo la generación total de residuos por ciudad (Marcó del Pont, 1997, pp. 36-38).

En 2004 se desarrolló un modelo matemático lineal multivariable para estimar la generación de RSU en el municipio de Nicolás Romero, Estado de México. Para lograrlo, el autor siguió dos líneas principales: 1) la información proporcionada por bases de datos públicas sobre ciertas variables socioeconómicas (independientes) en viviendas y unidades económicas, seleccionadas mediante la técnica de pasos hacia atrás para determinar su influencia sobre 2) la generación de RSU por localidad, como variable dependiente a través de la medición por pesos y sus tiempos de generación (Rodríguez, 2004, pp. 47-49).

Cabe señalar que la presente investigación se centra en los RO generados en las UECS, entre las que se incluyen: restaurantes, mercados, venta de alimentos, tiendas de autoservicio, hoteles, centros educativos y establecimientos recreativos. Este trabajo parte de la hipótesis de que el aumento en la generación de los RSU (en particular los RO), en la CDMX se presenta en el sector domiciliar y también en las UECS (Buenrostro et al., 2001, p. 88; Rodríguez, 2004, p. 47; Intharathirat et al., 2015, p. 5).

Así, los objetivos propuestos para este trabajo son: $i$ ) actualizar la estimación de los RO en los RSU en la CDMX aportados por las UECS, mediante un método alternativo basado en datos demográficos, y ii) identificar los factores que influyen en dicha generación. Para ello, este estudio pretende reestablecer las estimaciones mediante la aplicación de las tasas de generación de RSU/d por el tipo y número de empleados registrados en las UECS, cuyos índices se renuevan constantemente en el DENUE (INEGI, 2016a), tal como se hizo en el trabajo de Purcell y Magette $(2009$, p. 3) para la ciudad de Dublín y se propone en el Diagnóstico Básico para la Gestión Integral de Residuos (INECC, 2012, p. 60). Posteriormente, a los resultados obtenidos se les aplicaron las proporciones encontradas para la generación de RO por tipo de UECS, según el estudio realizado en la CDMX por la Agencia de Cooperación Internacional Japonesa (JICA) y el gobierno local, mismas que fueron proyectadas hasta el año 2010 (JICA-GDF, 1999, p. 45).

También se corroboró la relación entre el número de UECS y la generación de RO obtenida, con el fin de identificar los factores que influyen en sus variaciones de producción, mediante un modelo predictivo, y emplearlo como parte metodológica en programas futuros. 


\section{Métodos}

\subsection{Sitio de estudio}

La CDMX se ubica geográficamente entre las latitudes $19^{\circ} 36^{\prime} \mathrm{N}$ y $19^{\circ} 03^{\prime}$ $\mathrm{N}$ y entre las longitudes $98^{\circ} 57^{\prime} \mathrm{W}$ y $99^{\circ} 22^{\prime} \mathrm{W}$ (INEGI, 2016, p. 1.1). De acuerdo con la última encuesta intercensal, la ciudad cuenta con 8918653 de habitantes en un área de $1490 \mathrm{~km}^{2}$ (INEGI, 2015). Esta capital está conformada por 16 delegaciones políticas, en donde se ubican las UECS, de acuerdo con el registro del DENUE (INEGI, 2016a).

\subsection{Aplicación de las tasas de generación de residuos sólidos urbanos por empleado al registro actualizado del DENUE}

De acuerdo con la propuesta de Lebersorger y Beigl (2011), se recopiló la información estadística sobre las UECS disponible en la página del Instituto Nacional de Estadística y Geografía (INEGI). Para calcular las tasas de generación de RSU por tipo de UECS, se recurrió al método empleado por Purcell y Maguete (2009, pp. 8-9) para la ciudad de Dublín. En el caso de la CDMX, se emplearon las tasas de generación por empleado o huésped o alumno, ofrecidas por el estudio de JICA-GDF (1999, p. 45), cuyos resultados eran detallados y fueron proyectados hasta el año 2010, así como el informe realizado por la Dirección General de Servicios Urbanos de la Sobse (INE, 2007, pp. 2-3). Las tasas de generación de RSU se presentan en el Cuadro 2.

Las tasas de generación de RSU identificadas por tipo de UECS, fueron aplicadas entonces al número promedio de empleados registrados en el DENUE hasta inicios de 2016, presentados por el INEGI (2016a), el cual contiene a las Unidades Económicas clasificadas por actividades económicas, de acuerdo con los sectores del Sistema de Clasificación Industrial de América del Norte (INEGI, 2013a, p. 17). En el DENUE la información se presenta por tipo de negocio según su actividad, por el personal promedio ocupado y por su ubicación geográfica, incluyendo el nivel delegacional.

Para este trabajo se consideraron nueve tipos de UECS como potenciales generadores de RO por la actividad que desempeñaban:

a) Servicios de preparación de alimentos y bebidas (restaurantes, bares, cafeterías, fondas, comida rápida, etc.).

b) Servicios de alojamiento temporal (hoteles, moteles, casas de huéspedes, albergues, etc.). 


\section{Cuadro 2}

Tasas de generación de RSU en UECS para la Ciudad de México. Periodo 2007-2010

\begin{tabular}{|c|c|}
\hline Sector & Tasa generada de $R S U$ \\
\hline Tienda de autoservicio & $\begin{array}{l}637000 \mathrm{~kg} / \text { establecimiento / día } \\
2.527 \mathrm{~kg} / \text { empleado / día }\end{array}$ \\
\hline $\begin{array}{l}\text { Tienda departamental con } \\
\text { restaurante }\end{array}$ & $\begin{array}{l}368000 \mathrm{~kg} \text { / establecimiento / día } \\
1.468 \mathrm{~kg} / \text { empleado / día }\end{array}$ \\
\hline $\begin{array}{l}\text { Tienda departamental sin } \\
\text { restaurante }\end{array}$ & $\begin{array}{l}368000 \mathrm{~kg} \text { / establecimiento / día } \\
0.766 \mathrm{~kg} \text { / empleado / día }\end{array}$ \\
\hline Local comercial & $\begin{array}{l}6.650 \mathrm{~kg} \text { / empleado / día } \\
2.875 \mathrm{~kg} \text { / empleado / día }\end{array}$ \\
\hline Mercado sobre ruedas o Tianguis & $\begin{array}{l}575800 \mathrm{~kg} / \text { tianguis / día } \\
2.758 \mathrm{~kg} / \text { local }^{\text {a }} \text { día }\end{array}$ \\
\hline Mercado especializado & $3.350 \mathrm{~kg} /$ local $^{\mathrm{a}} /$ día \\
\hline Mercado común & $2.153 \mathrm{~kg} /$ local $^{\mathrm{a}} /$ día \\
\hline Restaurantes y bares & $\begin{array}{l}25442 \mathrm{~kg} / \text { establecimiento / día } \\
0.850 \mathrm{~kg} / \text { comensal }^{\mathrm{b}} / \text { día }\end{array}$ \\
\hline Cines & $\begin{array}{l}1.230 \mathrm{~kg} / \text { empleado / día } \\
0.054 \mathrm{~kg} \text { / espectador / día }\end{array}$ \\
\hline Instalaciones deportivas & $\begin{array}{l}2.620 \mathrm{~kg} \text { / empleado / día } \\
0.054 \mathrm{~kg} / \text { espectador / día }\end{array}$ \\
\hline Centros culturales & $0.330 \mathrm{~kg} /$ empleado / día \\
\hline Hoteles 5 estrellas & $\begin{array}{l}1016900 \mathrm{~kg} / \text { establecimiento / día } \\
1.035 \mathrm{~kg} \text { / huésped / día }\end{array}$ \\
\hline Hoteles 4 estrellas & $\begin{array}{l}218500 \mathrm{~kg} / \text { establecimiento / día } \\
1.035 \mathrm{~kg} \text { / huésped / día }\end{array}$ \\
\hline Hoteles 3 estrellas & $\begin{array}{l}16810 \mathrm{~kg} \text { / establecimiento / día } \\
1.035 \mathrm{~kg} \text { / huésped / día }\end{array}$ \\
\hline Centro educativo preescolar & $0.040 \mathrm{~kg} /$ alumno / día \\
\hline Primaria & $0.055 \mathrm{~kg} /$ alumno ${ }^{\mathrm{c}} /$ día \\
\hline Capacitación para el trabajo & $0.060 \mathrm{~kg} /$ alumno ${ }^{\mathrm{c}} /$ día \\
\hline Secundaria & $0.065 \mathrm{~kg} /$ alumno $/$ día \\
\hline $\begin{array}{l}\text { Bachillerato } \\
\text { Técnico }\end{array}$ & $0.060 \mathrm{~kg} /$ alumno ${ }^{\mathrm{c}} /$ día \\
\hline Superior & $0.070 \mathrm{~kg} /$ alumno ${ }^{\mathrm{c}}$ / día \\
\hline
\end{tabular}

${ }^{a}$ El número promedio de empleados por local es 2 (Gayosso, 2009, p. 62).

${ }^{\text {b }}$ El número promedio de comensales por empleado es 20 por día (La Verdad, s / f).

${ }^{\text {c }}$ El número promedio de alumnos por empleado educativo es 25 (Sistema Nacional de Información Estadística Educativa, 2015-2016).

Fuente: JICA/GDF (1999, p. 42) y DGSU (INE, 2007, pp. 2-3). 
c) Establecimientos comerciales con venta al por menor de abarrotes, alimentos, bebidas, hielo y tabaco; aquí se incluyen los establecimientos presentes en mercados comunes o especializados.

d) Establecimientos comerciales con venta al por menor en tiendas de autoservicio y departamentales.

e) Establecimientos comerciales con venta al por mayor de abarrotes, alimentos, bebidas, hielo y tabaco.

f) Servicios educativos (escuelas de todos los niveles, de todo tipo, y relacionadas con el apoyo a este servicio).

g) Tianguis o mercados ambulantes sobre la vía pública.

h) Culturales, deportivas y de recreación.

A partir de los porcentajes de RO (alimentos y poda) ofrecidos por el estudio de JICA-GDF (1999, p. 45). En el Cuadro 3 se realizó el cálculo con los datos actualizados obtenidos del DENUE en la que se aprecia que los sectores de mercados y restaurantes contribuyen con la mayor proporción de este tipo de residuos en el sector alimenticio, mientras que en jardinería, son los centros educativos y hoteles.

\subsection{Preparación del modelo de estimación}

Para utilizar un modelo apropiado de las condiciones de generación de RO en UECS, como variable dependiente, se revisaron trabajos que utilizaron modelos para determinar volúmenes de residuos -fueran orgánicos o noproducidos en el sector comercial, así como sus variables independientes.

\section{Cuadro 3}

Porcentajes de RO e inorgánicos (RI) generados por tipo de UECS

\begin{tabular}{lccc}
\hline \multirow{2}{*}{ Tipo de UECS } & \multicolumn{2}{c}{ RO (\%) } & Inorgánicos \\
\cline { 2 - 3 } & Alimenticios & Jardinería & 61.12 \\
\hline Comercios & 38.73 & 0.15 & 36.87 \\
Mercados & 63.08 & 0.05 & 25.49 \\
Restaurantes & 74.43 & 0.08 & 83.41 \\
Centros recreativos & 16.17 & 0.420 & 53.11 \\
Hoteles & 43.23 & 3.66 & 77.66 \\
Centros Educativos & 16.02 & 6.32 & \\
\hline
\end{tabular}

Fuente: JICA/GDF (1999, p. 45) con proporciones proyectadas para el año 2010. 
Entre ellos destacaron el de Purcell y Magette (2009, p. 14), con RO comerciales y algunas variables socioeconómicas, y el más cercano en contexto, el de Rodríguez (2004, p. 29), que presentó un modelo matemático de generación de residuos residencial y para unidades económicas en un municipio que forma parte del área metropolitana de la CDMX.

Para identificar los factores que influyen en la generación de RO se empleó como técnica de análisis la regresión lineal múltiple, cuya fórmula se presenta a continuación:

$$
y=b 0+b 1 X 1+b 2 X 2+\ldots+b n X n+U
$$

donde $y$ representa la variable dependiente que proviene de la generación total de RO por UECS, por delegación. El coeficiente $b 0$ es la ordenada al origen o coeficiente independiente, los restantes coeficientes $b$ representan los cambios promedio en respuesta a su respectiva variable $X_{n}$, las cuales son llamadas independientes y provienen de las mismas variables socioeconómicas utilizadas por el trabajo de Rodríguez (2004, p. 52) para el mismo contexto de la CDMX. Entre ellas se encuentran el PIB, el número de UE, la producción bruta total, etc., que se volvieron a utilizar en este trabajo por haber sido ya validadas; sin embargo, se utilizaron en esta ocasión los datos actualizados del último Censo de Población y Vivienda del INEGI (2010). Finalmente, $U$ representa el error al azar promedio y que se espera sea igual a cero.

Las definiciones para las variables independientes de tipo escalar utilizadas provienen de la metodología presentada por el INEGI (2014b) y son las siguientes:

a) Producto Interno Bruto (PIB). Es la suma de los valores monetarios de los bienes y servicios producidos por delegación. Unidad: miles de pesos.

b) Unidad Económica Comercial y de Servicio (UECS). Es una entidad productora de bienes o servicios. Unidad: número de unidades.

c) Producción Bruta Total (PBT). Es el valor de todos los bienes y servicios producidos o comercializados por cada unidad económica como resultado del ejercicio de sus actividades. Unidad: miles de pesos.

d) Personal Ocupado (PO). Comprende a todas las personas que trabajaron durante el periodo de referencia. Unidad: número de empleados.

e) Activos Fijos Netos (AFN). Es el valor de todos aquellos bienes, 
propiedad de la unidad económica, que tienen la capacidad de producir bienes y servicios. Unidad: miles de pesos.

f) Valor Agregado Censal Bruto (VACB). Es el valor de la producción menos el valor del consumo intermedio antes de deducirse el consumo de capital fijo. Es una medida de la contribución al PIB hecha por una unidad de producción. Unidad: miles de pesos.

g) Remuneraciones (R). Es la retribución total, en dinero y/o en especie, que una unidad económica paga a los asalariados por la contraprestación del trabajo realizado durante el periodo contable. Unidad: miles de pesos.

El análisis estadístico fue realizado en el programa Statistical Package for the Social Sciences (SPSS), versión 21. Para determinar el ajuste del modelo, se consideraron los siguientes criterios, de acuerdo con Buenrostro y colaboradores (2001, p. 90):

1) En la porción de la variación explicada de la muestra $\left(\mathrm{R}^{2}\right)$, un valor cercano a 1 es considerado un modelo con buen ajuste;

2) Un valor más bajo de la media al cuadrado de la regresión significaba un mal ajuste;

3) El modelo con una $F$ menor a 0.05 será un valor significativo.

\section{Resultados y discusión}

\subsection{Estimación actualizada de la generación de residuos sólidos urbanos por Unidades Económicas Comerciales y de Servicios}

Resulta apropiado observar las tendencias que han tenido las UECS analizadas en este trabajo a lo largo del tiempo. El Cuadro 4 muestra que todos los sectores considerados están en constante aumento y que el sector de comercio al por menor y el de servicio de alojamiento temporal y preparación de alimentos y bebidas son los que han alcanzado un mayor crecimiento, aunque cabe destacar también el alza en el último año de los servicios educativos.

De acuerdo con el Cuadro 5, los resultados obtenidos por delegación, sobre la aplicación de las tasas de generación de RSU por empleado por día, según el número de empleados registrados en el DENUE a inicios de 2016, muestra que la generación estimada de RSU para la CDMX fue de 30565 t/d, cantidad que sobrepasa a la cifra oficial de $12893 \mathrm{t} / \mathrm{d}$ difundida en el 


\section{Cuadro 4}

Comportamiento de las UECS en la Ciudad de México, 2004-2016

\begin{tabular}{lrrrr}
\hline \multicolumn{1}{c}{ Actividad económica } & 2004 & 2009 & \multicolumn{1}{c}{$\mathbf{2 0 1 4}$} & \multicolumn{1}{c}{$\mathbf{2 0 1 6}$} \\
\hline Comercio al por mayor & 10935 & 12854 & 15698 & 17242 \\
Comercio al por menor & 170118 & 183439 & 194923 & 206589 \\
$\begin{array}{l}\text { Servicios educativos } \\
\begin{array}{l}\text { Servicios de esparcimiento } \\
\quad \text { cultural y deportivo }\end{array}\end{array}$ & 5103 & 5948 & 6347 & 11854 \\
$\begin{array}{l}\text { Servicios de alojamiento } \\
\text { temporal y de preparación } \\
\text { de alimentos y bebidas }\end{array}$ & 35235 & 41261 & 49322 & 54121 \\
\begin{tabular}{l} 
Total \\
\hline
\end{tabular} & 224701 & 247346 & 271027 & 295565 \\
\hline
\end{tabular}

Fuente: Censos económicos (2004; 2009; 2014), INEGI (2016a).

Inventario de Residuos Sólidos 2014, y de 12843 t/d en el correspondiente a 2015 (Sedema, 2014, p. 11; 2015, p. 11); se consideran todas las fuentes de generación.

Según el análisis del PGIRS 2004-2010, realizado por Wismer y Lopez de Alba (2011, p. 4), también se encontraron inconsistencias en los valores de la generación de RSU reportados para el año 2008, a través de comparar los datos de ingreso a las instalaciones de manejo de residuos y las cifras oficiales reportadas en los inventarios. La generación de residuos estimada fue de 14000 t/d, en tanto el PGIRS reportó $12000 \mathrm{t} / \mathrm{d}$ en el mismo año. Dicha investigación subraya la necesidad de contar con una metodología que sustentara los datos oficiales difundidos. A finales de 2013 se realizó un diagnóstico para actualizar el flujo de RSU en la CDMX (CIIEMAD-IPN, 2013, pp. 11-16). En éste se señalaron inconsistencias entre los datos de generación en el periodo 2011 a septiembre de 2013, aportados por las delegaciones (14 557.14 t/d) y por la Sobse (11 $500.30 \mathrm{t} / \mathrm{d})$. Por su parte, el Inventario de Residuos Sólidos 2013 reportó 12816 t/d (Sedema, 2014, p. 1). Estas incongruencias se explican por la carencia de una normatividad en el cálculo de la generación de residuos, ya que se limitan a estimaciones sin hacer pesajes en esta etapa del manejo (CIIEMAD-IPN, 2013, p. 87).

De acuerdo con los resultados de la presente investigación, las tres delegaciones que presentaron mayor generación de RSU en las UECS, fueron: Iztapalapa (16.7\%), Cuauhtémoc (12.3\%) y Gustavo A. Madero (10.1\%); en cambio, Cuajimalpa (2\%), La Magdalena Contreras (1.5\%) y Milpa Alta (1.3\%) reportaron los menores aportes. Según los inventarios de residuos sólidos 


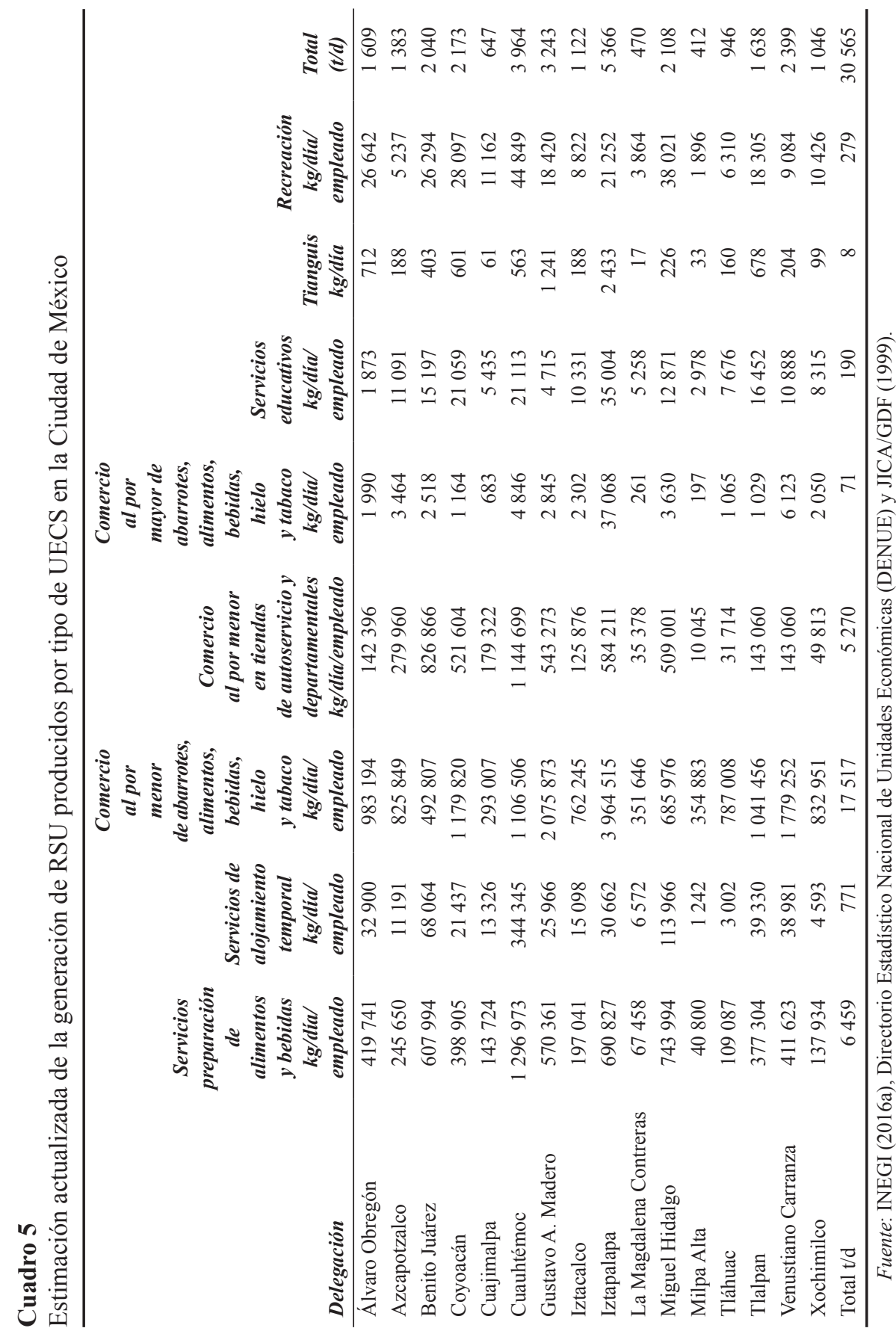


de 2014 y 2015, las delegaciones con mayor generación fueron: Iztapalapa (17.7\%), Gustavo A. Madero (13.3\%) y Cuauhtémoc (10.1\%); mientras que La Magdalena Contreras (2\%), Cuajimalpa (1.4\%) y Milpa Alta $(0.9 \%)$ contribuyeron con la menor proporción (Sedema, 2014, p. 12; 2015, p. 12).

El inventario 2015 (Sedema, 2016, p. 11) establece que la variabilidad en la generación de los residuos es una función de diversas variables, como: "el desarrollo tecnológico, industrial y económico, su densidad poblacional, hábitos de consumo, nivel de ingreso, conciencia ambiental, entre otras". A fin de definir cuáles variables podían influir en la dinámica de generación de RO, se recurrió al trabajo de Rodríguez (2004), que propone una serie de variables validadas acorde con la hipótesis de este trabajo, a saber, que el aumento de las UECS en la CDMX impacta en el aumento en la generación de los RSU, particularmente de los RO.

Para 2014 en la CDMX el total de UECS fue de 415481 (Censos Económicos, 2014) y para el segundo semestre del 2015 se registraron 460370 (INEGI, 2016a), lo que representó un aumento de 10\% en un año. En un estudio similar realizado en la ciudad de Dublín, Purcell y Magette (2009, p. 14), registraron 2261 unidades económicas comerciales con una generación estimada de 3523.5 toneladas de RO por semana, es decir, 500 t/d, lo cual indica que el número de UECS puede ser un factor que influye en la cantidad de generación de estos residuos, como lo menciona Grazhdani (2016, p. 11).

La Figura 2 muestra la tendencia de los sectores de las UECS con potencial de generación de RO, así como sus porcentajes de generación de RSU. El aumento de los giros dedicados a la preparación de alimentos (restaurantes, bares, etc.) llegó a 30\%, mientras que los de servicios de alojamiento temporal y los centros culturales y deportivos aumentaron menos de 5\% en los últimos seis años. Caso contrario, los comercios (incluyendo mercados y tianguis) se han mantenido, mientras que las escuelas redujeron su generación en menos de $2 \%$ respecto al periodo anterior.

\subsection{Estimación actualizada de la generación de residuos orgánicos a partir de las Unidades Económicas Comerciales y de Servicios}

De acuerdo con los resultados, el número total de UECS con potencial generador de RO, registradas en el Directorio DENUE a inicios del 2016, fueron 163 129; lo que representa $39.27 \%$ del total. Conforme a las proyecciones de JICA/GDF (1999, p. 45) para el año 2010, se aplicaron los porcentajes de las unidades t/año al total de UECS registradas al primer bimes- 


\section{Figura 2}

Comportamiento de los porcentajes de generación de RSU en las Unidades Económicas y de Servicios en la Ciudad de México, 1999-2016

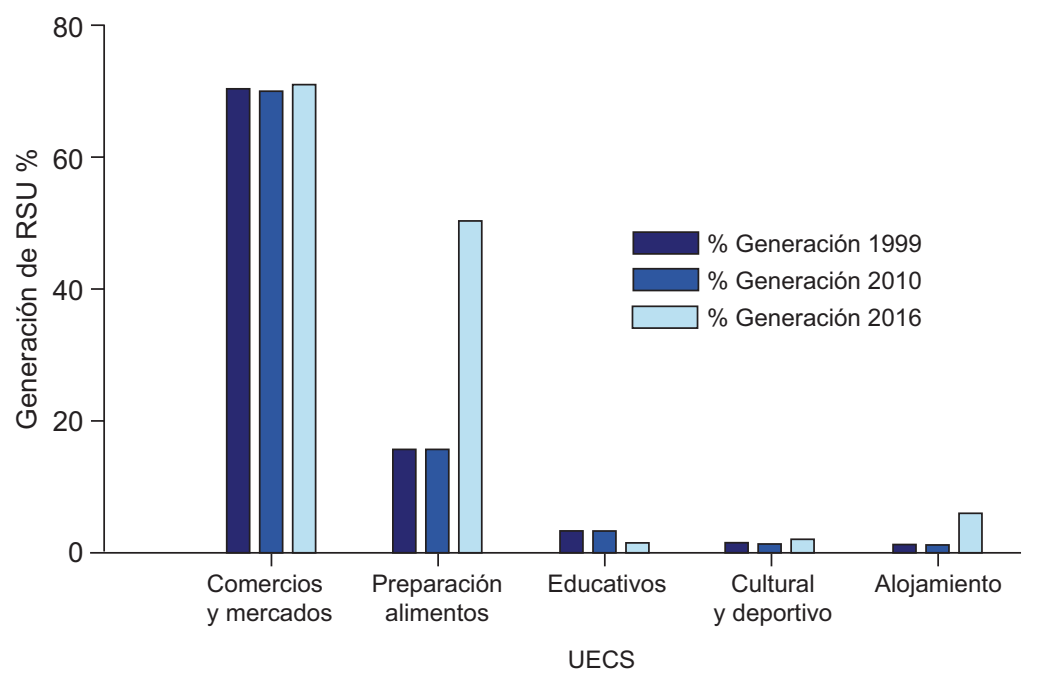

Fuente: JICA/GDF (1999, p. 44) proyecciones al 2010 y aplicación de tasas de generación de RSU con base en los registros del DENUE (INEGI, 2016a).

tre de 2016, para obtener una estimación de los RO generados para este trabajo, la cual fue de $14155 \mathrm{t} / \mathrm{d}$ para las 16 delegaciones de la capital.

Con el fin de identificar cuál era la aportación de RO en UECS por delegación, se presenta la Figura 3, donde se observa que las delegaciones Iztapalapa (7.6\%), Cuauhtémoc (6.6\%) y Gustavo A. Madero (4.8\%) aportan la mayor cantidad de RO por día, en contraposición a Cuajimalpa (1\%), La Magdalena Contreras $(0.7 \%)$ y Milpa Alta $(0.6 \%)$, con las menores proporciones.

Las tres delegaciones con más aportes a la generación de RO por UECS se presentan con mayor detalle en el Cuadro 6, donde se observa que en la delegación Iztapalapa el comercio de alimentos y bebidas, principalmente, ocupa hasta $70.2 \%$, seguido de las UECS encargadas de venta al por menor de alimentos y bebidas (21\%). En la delegación Cuauhtémoc el mayor porcentaje de las UECS está relacionado con la preparación de alimentos y bebidas (48\%), con el comercio al menudeo en 


\section{Figura 3}

Generación estimada de residuos orgánicos por delegación en la Ciudad de México, 2014-2016

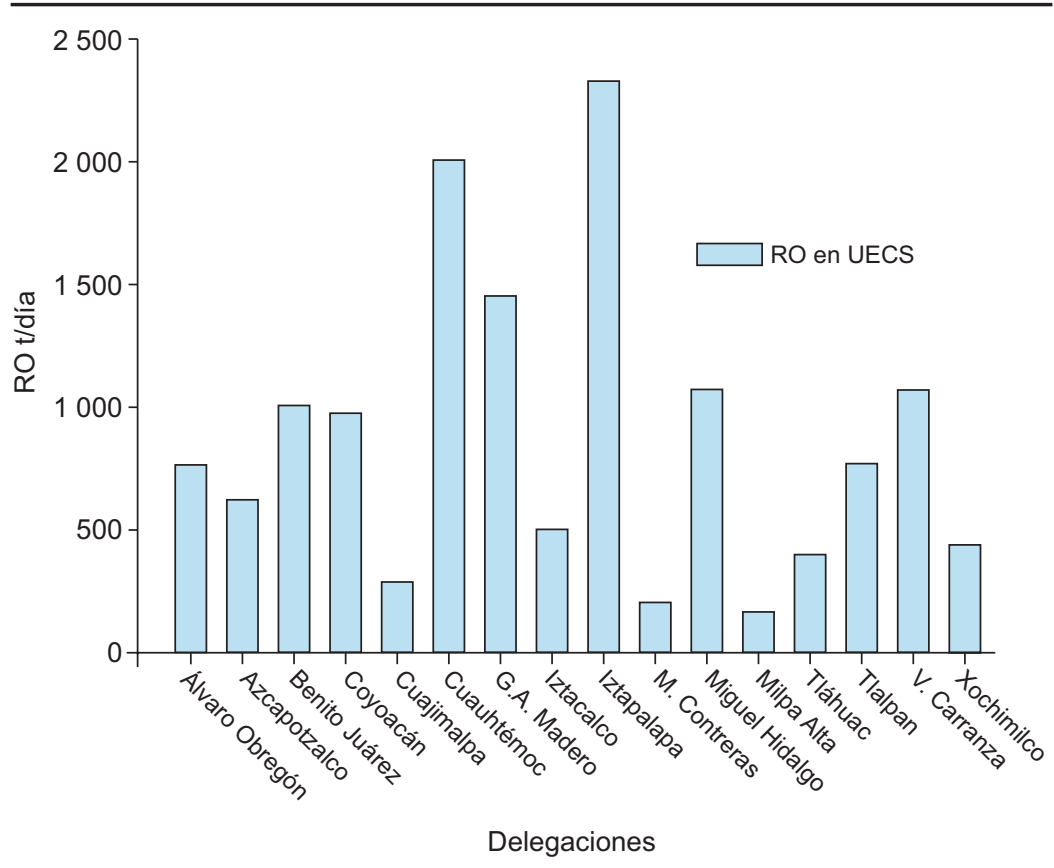

Fuente: Datos actualizados obtenidos de INEGI (2016a), Directorio Estadístico Nacional de Unidades Económicas (DENUE).

autoservicios (21.9\%) y con el de alimentos (21\%). En la delegación Gustavo A. Madero el comercio al menudeo de alimentos alcanza el 55\%, seguido de la preparación de alimentos $(29 \%)$ y el comercio al menudeo en tiendas de autoservicio (14.5\%).

Resulta ilustrativo comparar los resultados de este trabajo con los registros de recolección de RO en todas las delegaciones y el porcentaje de eficiencia en la separación de residuos reportada por las fuentes oficiales para observar si se considera la presencia y el crecimiento de las UECS. Para ello, se muestra la Figura 4, donde se observa que la generación de RO reportada por la Sedema (2014, p. 7) sobrepasa la cantidad de RSU recolectados en 2012 (INEGI, 2013, p. 6).

Esto es lógico ya que la recolección es una etapa posterior a la generación; no obstante, se esperaría que las tendencias pudieran comportarse si- 


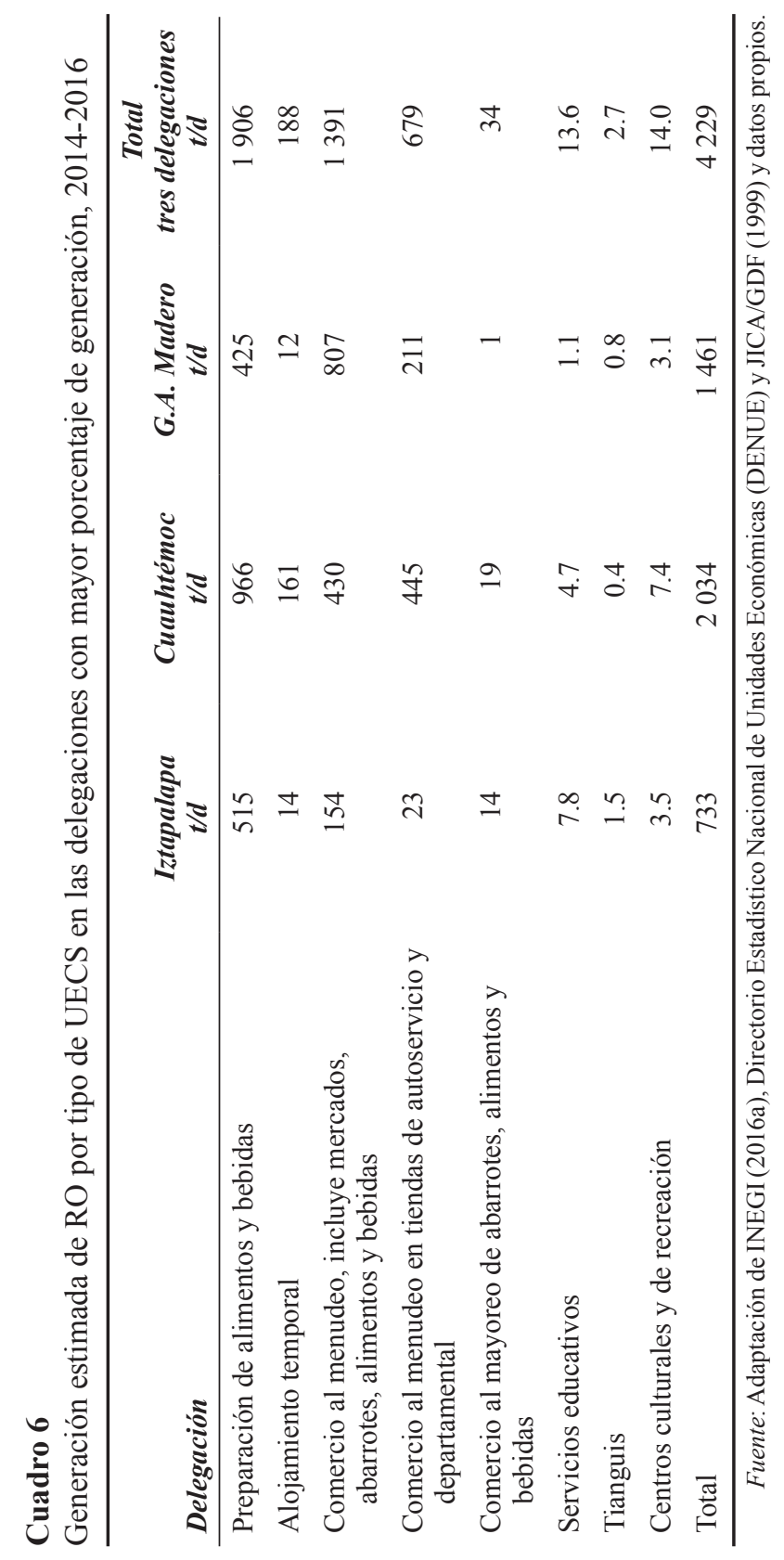


milarmente ya que, como se mencionó, en la recolección se cuenta con básculas en la salida de las estaciones de transferencia y plantas de selección (CIIEMAD-IPN, 2013, p. 87); sin embargo, a dichas estaciones no sólo llegan residuos generados en la CDMX, sino también del Estado de México, de particulares, etcétera.

Como se aprecia en la Figura 4, resulta paradójico que las delegaciones con mayor generación estimada de RO para este trabajo, son las que presentan los porcentajes más bajos de eficiencia en la separación, como Iztapalapa, Cuauhtémoc y G. A. Madero. Por otra parte, las delegaciones con menor generación como M. Contreras y Milpa Alta presentan las más altas tasas de eficiencia en la separación de residuos.

La deficiencia de una separación en fuente limita la posibilidad de pensar que, a pesar de que la población residencial, comercial o de servicios haya aumentado, la ciudad cuenta con un programa de reciclaje de residuos eficiente (pepena) por lo cual, la generación de RSU se pueda comportar de manera independiente al crecimiento poblacional, tal como lo propone el estudio de Grazhadni en Grecia (2016, p. 11).

\subsection{Modelo de estimación para la generación de residuos orgánicos en las Unidades Económicas Comerciales y de Servicios}

A fin de contar con un modelo de generación de RO de las UECS en la CDMX, se recurrió a las mismas variables independientes utilizadas por Rodríguez (2004, p. 52), entre ellas: PIB, número de UECS, PBT, PO, AFN, VACB y R, descritas previamente, cuyas cifras fueron actualizadas con los datos del último censo poblacional (INEGI, 2010), así como con el último Censo Económico para la Ciudad de México (INEGI, 2014).

En primer término, se utilizó como variable dependiente la cantidad total generada de RSU por las UECS ( $\mathrm{t} / \mathrm{d}$ ) obtenida en este trabajo. Todas las variables fueron aplicadas a una regresión lineal múltiple con pasos hacia atrás, para evitar la colinealidad entre variables independientes (Bello, 2016, p. 1). Los resultados tuvieron una $\mathrm{R}^{2}$ corregida de $98.6 \%$, es decir, ya estaba considerado el error típico de la estimación, y una $p=0.00$. El modelo estandarizado resultante fue el siguiente:

$$
\wedge_{z}=0.636 U E C S+0.45 P O
$$

que presentó una significación de 0.00 en el cambio en F. 


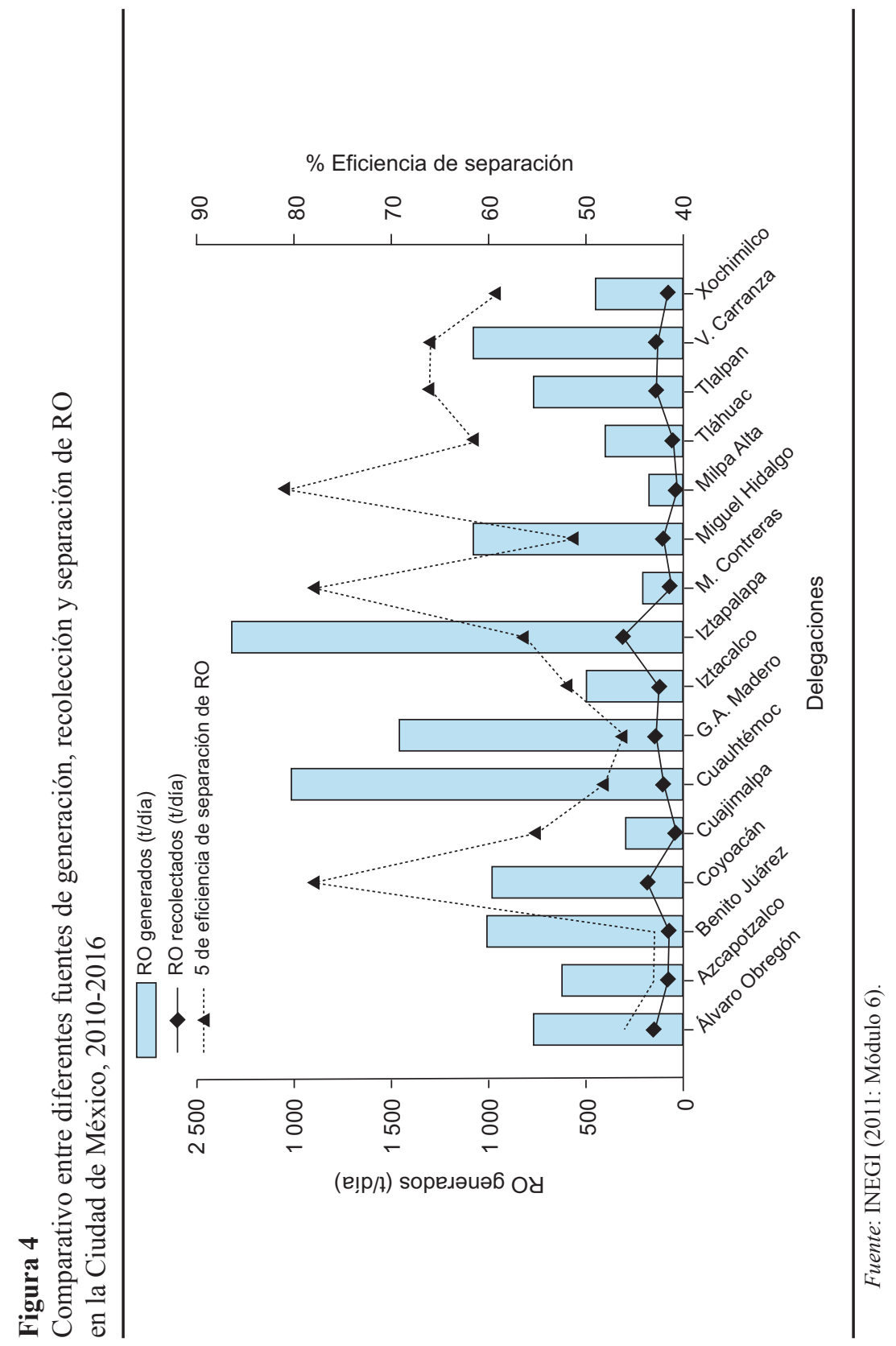


Posteriormente, se introdujo en otro modelo la cantidad total estimada de RO generados en las UECS como variable dependiente, y las independientes correspondieron a las cifras actualizadas de las variables del tipo económico únicamente, presentes en el trabajo de Rodríguez (2004, p. 52). Se aplicó la prueba de Shapiro-Wilk para probar la normalidad y para examinar la relación entre las variables, y se calculó nuevamente el análisis de regresión múltiple por pasos sucesivos. El modelo obtenido fue el siguiente:

$$
\wedge_{z}=0.807 U E C S+0.386 V A C G
$$

La ecuación presentó una $\mathrm{R}^{2}$ corregida de 0.961 , por lo que ya se encuentra expresada con los coeficientes estandarizados. El valor de Durbin Watson (1.409) indicó ausencia de problemas de correlación. Por otra parte, la prueba $\mathrm{F}$ global de medias fue significativa $(p=0.0001)$, con un valor de 183.7, lo que hace suponer que existe un efecto real de las variables independientes sobre la variable dependiente (generación de RO de las UECS).

Las variables presentes en el modelo obtenido, por orden de importancia, fueron el número de UECS con un valor de cambio de 0.807 $(t=15.789, p=0.00)$, seguida por la variable VACG con un valor de 0.386 $(t=7.266, p=0.00)$, ambas con valor de significación de 0.0001. El coeficiente $b 0 \mathrm{u}$ ordenada al origen de la Ecuación 1, fue retirado ya que no mostró un valor significativo en la prueba $t$ de los coeficientes tipificados.

La interpretación para este modelo sugiere que manteniendo la variable VACG sin cambios, la cantidad en promedio de RO generados aumentará $80.07 \mathrm{~kg} / \mathrm{d}$ cuando se agrega cada UECS a la Ciudad de México y, por otra parte, si se mantiene la variable UECS fija, significa que los RO aumentarán $0.386 \mathrm{~kg} / \mathrm{d}$ por cada mil pesos del valor agregado censal bruto.

De acuerdo con los resultados obtenidos por el modelo predictivo de Rodríguez (2004, p. 56), dentro del subsistema económico para el municipio conurbado a la Ciudad de México, las variables independientes que tuvieron los más altos valores de correlación fueron las unidades económicas totales (UECS), la producción total (valor de bienes y servicios producidos por las unidades económicas) y el personal ocupado (PO). De estas tres variables, el número de UECS es la que se repite en los resultados de este trabajo, pero a diferencia del modelo de Rodríguez (2004, p 56), aparece la variable valor agregado censal bruto (VACG), es decir, el valor económico generado por el conjunto de productores, que se añade a un bien después de haber pasado una o varias etapas de producción.

Finalmente, la variable del personal ocupado desaparece del modelo manejado en este trabajo. Ello debido, probablemente, a que el anterior se 
enfocaba en la generación de RSU totales, a diferencia de éste, donde únicamente se consideran los RO.

En el estudio de Grazhdani realizado en 15 poblaciones griegas, con resultados obtenidos mediante pruebas similares, se menciona que los centros comerciales más antiguos, donde hay una alta densidad de empleados, contribuyen de manera importante a la generación de residuos per cápita, por lo que este indicador podría reflejar una variable que el autor omitió por tener una alta correlación con el tamaño poblacional: el número de empleados. Con esta variable fuera, Grahzhdani obtuvo evidencia empírica necesaria para mostrar que el aumento en la generación de residuos era independiente del crecimiento poblacional (Grazhdani, 2016, p. 11).

\subsection{Importancia de los métodos de estimación en la generación de residuos}

La generación y el manejo de los RSU y los RME son un asunto de interés mundial, ya que representan un problema socioambiental complejo y son el resultado de un ciclo de producción y consumo (Ojeda-Benítez et al., 2008, p. S7). Es así que la política de residuos sólidos para la CDMX, dirigida hacia la prevención y la valorización en el manejo, responde al incremento en su generación y a sus impactos negativos en la salud pública, el ambiente y los recursos naturales. Esto es, reducir la generación en cada etapa del manejo de residuos en el marco de la gestión integral, entendida ésta como el conjunto articulado de acciones y lineamientos operativos, financieros, de planeación, administrativos, sociales, educativas, de monitoreo, supervisión y evaluación para el manejo de los residuos sólidos, desde su generación hasta su disposición final (LRSDF, 2003, art. 3 , fracc. XV111). Por tanto, resulta de gran importancia que dichas acciones y normas estén sólidamente fundamentadas para el logro de la política pública.

En este contexto, los modelos de estimación de residuos son fundamentales, ya que permiten conocer la generación en un momento específico y hacer proyecciones para conocer las necesidades futuras. Sin embargo, también requieren de datos confiables y actualizados para conocer la evolución histórica y la caracterización de los residuos generados.

En el marco de las políticas públicas, estos modelos constituyen una herramienta útil al aportar datos que permiten diseñar mejores instrumentos de política (programas públicos y sus acciones), evaluar su implementación y tomar decisiones para realizar los cambios necesarios, en su caso. 
Sin embargo, el contar con una política explícita en los documentos oficiales, la legislación, los arreglos político-administrativos y los programas, no es suficiente para lograr los objetivos de las políticas públicas. En la implementación se identifican gran cantidad de fallas, debido a que se ponen a prueba las decisiones políticas y en el proceso interactúan diversos actores (Aguilar, 2003, p. 26). Además, la implementación inicia cuando se han planteado los objetivos y metas de la política, después de que se ha formulado la legislación y se han destinado fondos para llevarla a cabo (Van Meter y Van Horn [1975] 2003, p. 100). En este contexto cabe subrayar la importancia de los recursos utilizados en la implementación: el poder, el personal, las finanzas, la información, la organización, el consenso, el tiempo, la infraestructura y el apoyo político, los cuales pueden variar dependiendo del tipo de política del que se trate (Knoepfel et al., 2007, pp. 64-65).

Para el caso que nos ocupa, si bien la LRSDF (2003), su Reglamento y el PGIRS establecen las responsabilidades para la Sedema, la Sobse y las delegaciones respecto a los inventarios de residuos, los planes de manejo, así como la revisión del PGIRS, estas acciones requieren una coordinación horizontal entre ellos, además de mecanismos de negociación y concertación. Así, la Sobse y las delegaciones tienen la mayor responsabilidad en el nivel operativo en el manejo de residuos y cumplen con el aporte de datos a la Sedema para la integración del inventario anual de residuos sólidos de la CDMX. Sin embargo, como se ha presentado en este trabajo, se carece de una metodología que fundamente los datos aportados por las entidades mencionadas.

Por otra parte, el programa rector de la política de residuos sólidos, el PGIRS 2016-2020, no establece las metas ni los objetivos para la reducción en la generación de residuos sólidos, con lo cual resulta poco viable conocer el avance en la implementación de la política encaminada a la valorización y a la minimización. De acuerdo con la eficiencia en la separación de RSU reportada en el último inventario de residuos 2015, no se ha alcanzado el objetivo primordial del PGIRS 2004-2009. Por lo tanto, resulta ambicioso plasmar en dicho Programa que se va a consolidar la gestión integral a través de un cambio en el paradigma y se tenderá a "Basura Cero" (PGIRS, 2016, p. 20). Más aún cuando se carece de los recursos financieros, de personal y tecnológicos para llevar a cabo la integración de los inventarios de residuos sólidos y de planes de manejo, y para el seguimiento y evaluación del PGIRS (Morales, 2016, p. 154). Como apunta Majone (1997, p. 195), las políticas tienen un carácter cambiante, en donde la parte estable de la política corresponde al núcleo, en tanto la periferia es la parte flexible, de tal manera que los programas particulares se mantienen en constante cambio, pero por lo general no afectan al núcleo. 
Por su parte, los RME son declarados por los generadores en los planes de manejo que presentan ante la Dirección General de Regulación Ambiental de la Sedema. De acuerdo con esto, existen alrededor de 400000 comercios y servicios registrados ( $97 \%$ son micro y pequeños establecimientos). En 2014 se ingresaron 5844 Licencias Ambientales Únicas (LAUDF), reportando un total de $1707.5 \mathrm{t} / \mathrm{d}$ de residuos (PGIRS, 2016, p. 10). Estas cifras vislumbran la tarea de la Sedema.

De esta manera queda patente la necesidad de realizar cambios en la coordinación de las entidades responsables de diseñar, ejecutar y evaluar la política de residuos sólidos en la Ciudad de México, así como de su programa rector. Esto a fin de consolidar las bases estadísticas de residuos sólidos, fundamentadas en una metodología unificada que permita la aplicación de modelos de estimación en la generación, y se aproveche así dicha herramienta para el logro de los objetivos de la política de residuos sólidos en la CDMX.

El planteamiento de este trabajo para estimar la generación de residuos por fuente, ofrece una alternativa diferente a la forma en que se ha venido haciendo, ya que se basa en dos líneas complementarias: 1) utilizar un procedimiento con sustento demográfico, mediante una base de datos de libre acceso y constante actualización, semejante a lo propuesto en el Diagnóstico básico para la gestión integral de residuos (INECC, 2012, p. 58); y 2) retomar las estimaciones obtenidas por la aplicación de la NMXAA-61-1985 (DOF, 2013a) por fuente de generación, como en trabajos anteriores, pero esta vez con datos actualizados.

De esta manera, se proporciona una reformulación al procedimiento utilizado convencionalmente, donde se espera contribuir con la estructuración de programas estratégicos para el control de la estandarización y actualización de la información existente, ya que tiene como base el uso compartido de variables socioeconómicas junto con las de índole escalar, en vista de la carencia de recursos de distintos tipos que aquejan al gobierno local. Con esto a la vista, se espera obtener una estimación más fidedigna para una mejor definición en los requerimientos de equipo, la inversión para transporte, recolección y disposición final y, por tanto, para la determinación de la carga potencial de contaminantes generados por delegación (INE, 1997).

Aunque el enfoque principal se hace hacia la generación de los RO provenientes de ciertos sectores económicos y de servicios de la CDMX, este trabajo deja entrever, para una futura discusión, la generación de los RI de estas mismas fuentes (Purcell y Maguette, 2011, p. 2180). De igual manera, un punto importante que no fue examinado corresponde a los RO provenientes de parques y jardines, debido a que la clasificación por fuente que presenta la DGSU (JICA-GDF,1999, p. 9) menciona que estos residuos 
pertenecen a vialidades y áreas verdes, aspectos no contemplados en la presente investigación.

Así, se abren oportunidades diferentes para el tratamiento y control de estos residuos, como su designación de manejo especial, lo cual les confiere características propias otorgadas por la LGPGIR (DOF, 2003) y la NOM161-Semarnat-1985 (DOF, 2013a), donde es la Federación la encargada de su manejo, o bien el gobierno local a través de un plan de manejo, dependiendo del volumen generado (PGIRS, 2004, p. 40).

\section{Conclusiones}

La información obtenida de este modelo de generación de los RO para las UECS en la CDMX, ofrece nuevas alternativas para la actualización y el enfoque sobre las variables relacionadas con la generación de este tipo de residuos, ya que está basada en aspectos demográficos actuales, lo cual también acelera la disponibilidad de contar con un método normalizado y renovado para la estimación de la generación de dichos residuos, así como de un nuevo estudio sobre las tasas de generación per cápita por fuente.

La idea de realizar estimaciones de la generación de este tipo de residuos a través de un método alternativo, basado en cifras demográficas a diferencia del utilizado normalmente, busca promover la reflexión y la inquietud sobre si los métodos tradicionales, basados únicamente en las estimaciones de la recolección provistos por la Sobse y las delegaciones, todavía tienen la capacidad de responder a las necesidades actuales y futuras sobre el manejo de residuos en la CDMX. Cuestión que plantea, a su vez, averiguar cuáles son los factores socioeconómicos que influyen en la generación de estos residuos, con el fin de acotar la labor de búsqueda y precisar el tipo de variables en las que se pueden enfocar los análisis para tener un monitoreo más accesible a falta de herramientas o recursos necesarios.

Gracias al análisis estadístico de las variables económicas con valores actualizados, se pudo determinar el papel del crecimiento de las UECS en la generación de los RSU, y por tanto, de los RO en la CDMX.

Como los resultados de este trabajo apuntan a que la generación de RO producidos por los sectores comerciales y de servicios en la capital del país es de $14155 \mathrm{t} / \mathrm{d}$, es importante reforzar las acciones de prevención en el PGIRS 2016. Entre éstas, la prevención en la generación de residuos de alimentos, y la valorización de proyectos para la generación de energía eléctrica, ecotecnologías domésticas, comerciales e institucionales que promuevan el compostaje y su aplicación. Asimismo, hay que fomentar la 
donación de alimentos en buen estado para comunidades con carencia alimentaria.

De igual manera, es importante redirigir los esfuerzos y recursos hacia un método normalizado en la forma de estimar la generación de RSU, con el fin de manejar cifras confiables y consistentes a lo largo de todas las etapas del manejo de residuos; de tal manera que los datos oficiales suministrados fundamenten una planeación adecuada para la gestión integral de los residuos, la cual permita, en primer término, proteger la salud pública y encaminar las acciones de forma ordenada y conjunta hacia una sustentabilidad más acorde con la realidad a la que se va enfrentando día con día la CDMX.

En el marco de las políticas públicas, queda patente la importancia de los actores político-administrativos, así como de los recursos disponibles en la implementación de la política de residuos sólidos de la CDMX. Esto sugiere la necesidad de evaluar y fortalecer el trabajo interinstitucional de las entidades encargadas de integrar los inventarios de residuos, de planes de manejo, así como de aquellos que aportan la información requerida. También alude a la importancia de revisar y evaluar el programa rector, como un instrumento de política, a fin de plasmar objetivos y metas realistas con base en la gestión integral.

\section{Bibliografía}

AGU (2017). Dirección de Transferencia y Disposición Final de los RSU, Agencia de Gestión Urbana. Ciudad de México. Recuperado de http://www.agu.cdmx. gob.mx/secretaria/estructura/91

Aguilar, Luis (2003). Estudio introductorio. En Luis Aguilar (coord.), La implementación de las políticas (pp. 15-92). México: Miguel Ángel Porrúa.

Bello, P.L.D. (2016). Regresión lineal múltiple. Métodos cuantitativos. Antioquia, Colombia: F.N.S.P. / Universidad de Antioquia.

Buenrostro, Otoniel, Bocco, Gerardo y Vence, Javier (2001). Forecasting generation of urban solid waste in developing countries. A case study in Mexico, Journal of the Air and Waste Management Association, 51(1), 86-93. Recuperado de http://www.tandfonline.com/doi/pdf/10.1080/10473289.2001.10464258

Castillo, Manuel Ángel (2003). La política de inmigración en México: un breve recuento. En Manuel Ángel Castillo, Alfredo Lattes y Jorge Santibáñez (coords.), Migración y fronteras (pp. 425-451). Tijuana: El Colegio de la Frontera Norte / Asociación Latinoamericana de Sociología / El Colegio de México, A.C.

Censos Económicos (2004). México: Sistema Automatizado de Información Censal. México: Instituto Nacional de Estadística y Geografía. Recuperado de http:// www.beta.inegi.org.mx/proyectos/ce/2004/ 
Censos Económicos (2009). México: Sistema Automatizado de Información Censal. México: Instituto Nacional de Estadística y Geografía. Recuperado de http:// www.beta.inegi.org.mx/proyectos/ce/2009/

Censos Económicos (2014). México: Sistema Automatizado de Información Censal México: Instituto Nacional de Estadística y Geografía. Recuperado de http:// www.beta.inegi.org.mx/proyectos/ce/2014/

CIIEMAD-IPN (2013). Diagnóstico actual del flujo de residuos sólidos urbanos que se genera en el Distrito Federal. México: Centro de Interdisciplinario de Investigaciones y Estudios sobre Medio Ambiente y Desarrollo / Procuraduría Ambiental y del Ordenamiento Territorial del Distrito Federal.

DOF (2003). Ley General para la Prevención y Gestión Integral de los Residuos. Diario Oficial de la Federación. México: Gobierno de la República. Recuperado de http://dof.gob.mx/nota_to_doc.php?codnota=688657

DOF (2013). Plan Nacional de Desarrollo 2013-2018. Diario Oficial de la Federación. México: Gobierno de la República. Recuperado de http://www.dof.gob.mx/ nota_detalle.php?codigo $=5299465$ yfecha $=20 / 05 / 2013$

DOF (2013a). NOM-161-Semarnat-2011 que establece los criterios para clasificar a los residuos de manejo especial y determinar cuáles están sujetos a plan de manejo; el listado de los mismos, el procedimiento para la inclusión o exclusión a dicho listado; así como los elementos y procedimientos para la formulación de los planes de manejo. Diario Oficial de la Federación. México. Recuperado de http://www.dof.gob.mx/nota_detalle.php?codigo=5286505yfecha=01/02/2013

FAO (2002). Declaración inaugural del Exmo. Sr. Kofi Annan. Ponencia presentada en la Reunión Internacional Informe de la Cumbre Mundial sobre la Alimentación, Organización de las Naciones Unidas para la Agricultura y la Alimentación, Roma, 10 a 13 de junio.

Garza, Gustavo (2003). La urbanización de México en el siglo XX. México: El Colegio de México, A.C.

Gayosso, Ramírez, José Luis, (2009). Los tianguistas de la Ciudad de México: de informales a trabajadores atípicos. Iztapalapa. Revista de Ciencias Sociales y Humanidades, 66, 53-67. Recuperado de http://www.redalyc.org/articulo. oa? id=39348723004

Gobierno del Distrito Federal (2004). Programa de Gestión Integral de los Residuos Sólidos, 2004-2009. Gaceta Oficial del Distrito Federal, 101-bis, $1^{\circ}$ de octubre. México. Recuperado de http://www.paot.org.mx/centro/programas/PGIRS. $\mathrm{pdf}$ ? $=$ po

Gobierno del Distrito Federal (2010). Programa de Gestión Integral de los Residuos Sólidos, 2010-2015. Gaceta Oficial del Distrito Federal, 925, 13 de septiembre. México.

GODF (2017). Ley de huertos urbanos en la Ciudad de México. Gaceta Oficial del Distrito Federal, 16 de febrero. México.

Grazhdani, D. (2016). Assessing the variables affecting on the rate of solid waste generation and recycling: An empirical analysis in Prespa Park. Waste Mana- 
gement, 48, 3-13. Recuperado de https:/www.ncbi.nlm.nih.gov/pubmed/ 26482808

INE (1997). Estadisticas e indicadores de inversión sobre residuos sólidos municipales en los principales centros urbanos de México. México: Instituto Nacional de Ecología.

INE (2007). Criterios técnicos para el diseño de sistemas de almacenamiento de residuos sólidos por tipo de fuente generadora. México: Instituto Nacional de Ecología.

INECC (2012). Diagnóstico básico para la gestión integral de residuos. México: Instituto Nacional de Ecología y Cambio Climático, Semarnat.

INEGI (2000). XII Censo General de Población y Vivienda 2000. México: Instituto Nacional de Estadística y Geografía. Recuperado de http://www.inegi.org.mx/ est/contenidos/Proyectos/ccpv/cpv2000/default.aspx

INEGI (2002). Estadísticas del medio ambiente del Distrito Federal y Zona Metropolitana, México: Instituto Nacional de Estadística y Geografía.

INEGI (2005). II Conteo de Población y Vivienda 2005, México: Instituto Nacional de Estadística y Geografía. Recuperado de http://www.inegi.org.mx/est/contenidos/ proyectos/ccpv/cpv2005/Default.aspx

INEGI (2006). Estadísticas del medio ambiente del Distrito Federal y Zona Metropolitana. México: Instituto Nacional de Estadística y Geografía.

INEGI (2010). Censo de Población y Vivienda 2010. México: Instituto Nacional de Estadística y Geografía. Recuperado de http://www.beta.inegi.org.mx/proyectos/ ccpv/2010/default.html

INEGI (2011). Censo Nacional de Gobiernos Municipales y Delegacionales 2011. Módulo 6. Residuos sólidos urbanos. México: Instituto Nacional de Estadística y Geografía.

INEGI (2013). Censo Nacional de Gobiernos Municipales y Delegacionales 2012. Módulo 6 Residuos Sólidos Urbanos. México: Instituto Nacional de Estadística y Geografía. Recuperado de http://www3.inegi.org.mx/sistemas/sisept/default. aspx? $\mathrm{t}=$ mamb1153 ys=est $\mathrm{yc}=34142$

INEGI (2013a). Sistema de Clasificación Industrial de América del Norte, México (SCIAN 2013). México: Instituto Nacional de Estadística y Geografía. Recuperado de http:/www.inegi.org.mx/est/contenidos/proyectos/scian/presentacion.aspx

INEGI (2014). Censos Económicos 2014. Resultados definitivos. México: Instituto Nacional de Estadística y Geografía. Recuperado de http://www.inegi.org.mx/ est/contenidos/proyectos/ce/ce2014/default.aspx

INEGI (2014b). PIB y cuentas nacionales. México: Instituto Nacional de Estadística y Geografía. Recuperado de http://www.inegi.org.mx/est/contenidos/proyectos/ $\mathrm{cn} /$ pibe/tabulados.aspx

INEGI (2015). Encuesta Intercensal 2015. México: Instituto Nacional de Estadística y Geografía. México. Recuperado de http://www.beta.inegi.org.mx/proyectos/ enchogares/especiales/intercensal/default.html

INEGI (2016). Anuario estadístico y geográfico de la Ciudad de México 2016. Mé- 
xico: Instituto Nacional de Estadística y Geografía. Recuperado de http://www3. inegi.org.mx/sistemas/biblioteca/ficha.aspx?upc $=702825084318$

INEGI (2016a). Directorio Estadístico Nacional de Unidades Económicas (DENUE). México: Instituto Nacional de Estadística y Geografía. Recuperado de http:// www3.inegi.org.mx/sistemas/mapa/denue/default.aspx

Instituto de Investigaciones Legislativas del Senado de la República (2004). Nuevas energías renovables: una alternativa energética sustentable para México (análisis y propuesta). México: Senado de la República LIX Legislatura / UNAM, Centro de Investigación en Energía. Recuperado de http://xml.ier.unam.mx/xml/ se/pe/NUEVAS_ENERG_RENOV.pdf

Intharathirat, R., Salam, P.A., Kumar, S. y Untong, A. (2015). Forecasting of municipal solid waste quantity in a developing country using multivariate grey models. Waste Management, 39, 3-14. Recuperado de https://www.ncbi.nlm.nih.gov/ pubmed/25704925

JICA/GDF (1999). Estudio sobre el manejo de residuos sólidos_para la Ciudad de México de los Estados Unidos Mexicanos. Informe final, volumen 1. México: Agencia de Cooperación Internacional de Japón / Gobierno del Distrito Federal. Recuperado de http://www2.df.gob.mx/secretarias/obras/servurbanos/estudio residuos.pdf

Jiménez, C. B. E. (2001). La contaminación ambiental en México. México: Limusa / Noriega.

Knoepfel, Peter, Laurre, Corinne, Varone, Frédic y Hill, Michael (2007). Public policy analysis. Reino Unido: Policy Press.

La Verdad (s/f). ¿Cuántos comensales/mesas atiende un camarero en un restaurante y en un bar tipo barra? España. Recuperado de http:/gastronomia.laverdad.es/ preguntas/alimentos/cuantos-comensales-mesas-atiende-camarero-restaurantebar-tipo-barra-11064.html (consulta: 7 marzo de 2017).

Lebersorger, S. y Beigl, P. (2011). Municipal solid waste generation in municipalities: Quantifying impacts of household structure, commercial waste and domestic fuel, Waste Management, 31, 1907-1915. Recuperado de https://doi.org/10.1016/ j.wasman.2011.05.016

Lebersorger, S. y Schneider, F. (2014). Food loss rates at the food retail, influencing factors and reasons as a basis for waste prevention measures, Waste Management, 34, 1911-1919. Recuperado de https://www.ncbi.nlm.nih.gov/pubmed/25060676

LRSDF (2003). Ley de Residuos Sólidos del Distrito Federal, México. Gaceta Oficial del Distrito Federal. Recuperado de http://aldf.gob.mx/archivo-b0980c936d0f7b 1b6138d5c613a6188c.pdf

Majone, Giandomenico (1997). Evidencia, argumentación y persuasión en la formulación de políticas. México: Fondo de Cultura Económica. [Estudio introductorio de Luis Aguilar].

Marcó del Pont, R. (coord.) (1997). Estadísticas e indicadores de inversión sobre residuos sólidos municipales en los principales centros urbanos de México. México: Semarnap, INE. 
Miezah, K., Obiri Danso, K., Kádár, Z., Fei-Baffoe, B. y Mensah, M.Y. (2015). Municipal solid waste characterization and quantification as measure towards effective waste management in Ghana. Waste Management, 46, 15-27. Recuperado de https://www.ncbi.nlm.nih.gov/pubmed/26421480

Monreal, D. (2015). Iniciativa estímulos fiscales para energías renovables. México. Recuperado de http://davidmonreal.mx/iniciativa-estimulos-fiscales-paraenergias-renovables/

Morales, Rosalba (2011). Planes de manejo de residuos de generadores de alto volumen: el caso de la Central de Abasto del Distrito Federal. Tesis de maestría en Ciencias en Estudios Ambientales y de la Sustentabilidad, Instituto Politécnico Nacional, Centro Interdisciplinario de Investigaciones y Estudios sobre Medio Ambiente y Desarrollo, México.

Morales, Rosalba (2016). Retos para la implementación de la politica de residuos sólidos en los mercados públicos de la Ciudad de México. Tesis de doctorado en Ciencias en Medio Ambiente y Desarrollo, Instituto Politécnico Nacional, Centro Interdisciplinario de Investigaciones y Estudios sobre Medio Ambiente y Desarrollo, México.

Ojeda-Benítez, S. Lozano-Olvera, G. Morelos, R. A., Armijo de Vega, C. (2008). Mathematical modeling to predict residential solid waste generation. Waste Management, 28, S7-S13. Recuperado de https://www.ncbi.nlm.nih.gov/pubmed/

OPS-OMS (1996). Análisis sectorial de residuos sólidos en México. Plan Regional de Inversiones en Ambiente y Salud. México: Organización Panamericana de la Salud, Organización Mundial de la Salud (Estudios, 10). Recuperado de http:// www.bvsde.paho.org/eswww/fulltext/analisis/mexico/mexico.html

PGIRS (2004). Acuerdo por el que se aprueba y expide el Programa General de Gestión Integral de Residuos Sólidos. Distrito Federal: Gobierno del Distrito Federal.

PGIRS (2010). Acuerdo por el que se aprueba y expide el Programa de Gestión Integral de Residuos Sólidos para el Distrito Federal, Gobierno del Distrito Federal.

PGIRS (2016). Acuerdo por el que se aprueba y expide Programa de Gestión Integral de Residuos Sólidos para la Ciudad de México, 2016-2020. Ciudad de México: Jefatura de Gobierno.

Purcell, M. y Magette, W. L. (2009). Prediction of household and commercial BMW generation according to socio-economic and other factors for the Dublin region. Waste Management, 29(4), 1237-1250. Recuperado de https://www.ncbi.nlm. nih.gov/pubmed/

Purcell, M. y Magette, W. L. (2011). Targeted intervention strategies to optimize diversion of BMW in the Dublin, Ireland region, Waste Management, 31, 21802189. Recuperado de https://doi.org/10.1016/j.wasman.2011.05.008

Reglamento de la LRSDF (2008). Reglamento de la Ley de Residuos Sólidos del Distrito Federal. Gaceta Oficial del Distrito Federal. México.

Rodríguez, S. (2004). Diseño de un modelo matemático de generación de residuos 
sólidos municipales en Nicolás Romero, México. Tesis de maestría, Instituto Politécnico Nacional, Centro Interdisciplinario de Investigación y Estudios sobre Medio Ambiente y Desarrollo, México.

Secofi (1985). Norma Mexicana NMX-AA-061-1985. Protección al ambiente. contaminación del suelo. Residuos sólidos municipales. Determinación de la generación. México: Secretaría de Comercio y Fomento Industrial. Recuperado de http://legismex.mty.itesm.mx/normas/aa/aa061.pdf

Sedema (2009). Inventario de Residuos Sólidos de la Ciudad de México 2008. México: Secretaría de Medio Ambiente del Gobierno del Distrito Federal.

Sedema (2012). Inventario de Residuos Sólidos de la Ciudad de México 2011. México: Secretaría de Medio Ambiente del Gobierno del Distrito Federal.

Sedema (2013). Inventario de Residuos Sólidos de la Ciudad de México 2012. México. Secretaría del Medio Ambiente del Distrito Federal.

Sedema (2013a). Norma Ambiental para el Distrito Federal NADF-024-AMBT-2013, que establece los criterios y especificaciones técnicas bajo los cuales se deberá realizar la separación, clasificación, recolección selectiva y almacenamiento de los residuos del Distrito Federal. México: Secretaría del Medio Ambiente del Distrito Federal.

Sedema (2014). Inventario de Residuos Sólidos de la Ciudad de México 2013. México: Secretaría del Medio Ambiente del Distrito Federal.

Sedema (2015). Inventario de Residuos Sólidos de la Ciudad de México 2014, México: Secretaría del Medio Ambiente del Distrito Federal.

Semarnat (2012). Informe de la situación del medio ambiente. Compendio de estadísticas ambientales. Indicadores clave y de desempeño ambiental. México: Secretaría de Medio Ambiente y Recursos Naturales.

Sistema Nacional de Información Estadística Educativa (2015-2016). Estadística del Sistema Educativo. Ciudad de México: Secretaría de Educación Pública. Recuperado de http://www.snie.sep.gob.mx/Estadistica.html

Sobrino, Jaime (2016). Localización industrial y concentración geográfica en México. Estudios Demográficos y Urbanos, 31(1), 9-56. Recuperado de http://estudios demograficosyurbanos.colmex.mx/index.php/edu/article/view/1502/1495

Tchobanoglous, G. y Kreith, F. (2002). Handbook of solid waste management. Estados Unidos, McGraw-Hill.

ONU-Habitat (2012). Estado de las ciudades de América Latina y el Caribe 2012. Rumbo a una nueva transición urbana. Río de Janeiro, Brasil: Programa de las Naciones Unidas para los Asentamientos Humanos. Recuperado de http://www. cinu.mx/minisitio/Informe_Ciudades/SOLACC_2012_web.pdf

Van Meter, Donald y Van Horn, Carl (2003). El proceso de implementación de las políticas. Un marco conceptual. En Luis F. Aguilar (ed.), La implementación de las políticas (pp. 97-146). México: Miguel Ángel Porrúa [trabajo originalmente publicado en 1975].

Wilson, D., Rodic, L., Cowing, M., Costas, A., Whiteman, A., Scheinberg, A., Vilches, R., Masterson, D., Stretz, J. y Oelz, B. (2015). "Wasteaware" benchmark indi- 
cators for integrated sustainable waste management in cities. Waste Management, 35, 329-342. Recuperado de https://doi.org/10.1016/j.wasman.2014. 10.006 Wismer, S. y Lopez de Alba, G. A. (2011). Evaluating the Mexican Federal District's Integrated Solid Waste Management Programme. Waste Management and Research, 29(5), 480-90. Recuperado de http://journals.sagepub.com/doi/ pdf/10.1177/0734242X10380493

\section{Acerca de las autoras}

Cecilia Esther Muñoz Cadena es bióloga general por la Universidad Autónoma Metropolitana, unidad Iztapalapa, con medalla al mérito universitario; maestra en Ciencias Ambientales (mención honorífica) por la Universidad Simón Bolívar, Ciudad de México, con la tesis Estudio comparativo de la producción de residuos sólidos urbanos inorgánicos callejeros (RSUIC) en dos colonias de la Ciudad de México; y doctora en Medio Ambiente y Desarrollo por el CIIEMAD, IPN, con la tesis Sistema de indicadores de la sustentabilidad socioambiental para los residuos sólidos urbanos en los espacios públicos de la Delegación Venustiano Carranza en el Distrito Federal, México.

Ha publicado artículos en revistas científicas nacionales e internacionales; entre ellos: Muñoz-Cadena, C. E., Estrada-Izquierdo, I. E. y MoralesPérez, R. E. (2016). Logros de la educación ambiental y la sustentabilidad urbana en México. Revista Electrónica de Investigación Educativa, 18(3), 37-50. Recuperado de http://redie.uabc.mx/redie/article/view/1192; MuñozCadena, C. E., Lina-Manjarrez, P., Estrada-Izquierdo, I. y Gallegos, E. (2012). An approach to litter generation and littering practices in a Mexico City neighborhood. Sustainability, 4, 1733-1754. doi:10.3390/su4081733; MuñozCadena, C. E., Lina-Manjarrez, P., Estrada-Izquierdo, I. y Bello, L. D. (2012). La técnica del árbol como estrategia de identificación de variables categóricas, aplicada al comportamiento de disposición inadecuada de residuos en los espacios públicos. Publicación electrónica por SINSIA-IPN.

Rosalba Esther Morales Pérez es ingeniera geóloga por la Facultad de Ingeniería de la UNAM; especialista en Hidrogeología por la Universidad Complutense de Madrid, España; maestra en Ciencias y Estudios Ambientales y de la Sustentabilidad (mención honorífica y reconocimiento al "Mejor desempeño académico de alumnos de posgrado 2012"), y doctora en Ciencias en Medio Ambiente y Desarrollo, por el CIIEMAD del IPN. Cuenta con más de 20 años de experiencia en los sectores público y privado. Sus 
áreas de interés son: gestión y política ambiental, residuos sólidos, mercados públicos y sustentabilidad. Ha publicado artículos en revistas científicas nacionales e internacionales, entre ellos: Morales-Pérez, R. y Mejía Ponce de León, A. (2016). La prevención de residuos de alimentos. Los casos de la Unión Europea y la Ciudad de México. Sociedad y Ambiente, 10, 78-94; Muñoz-Cadena, C. E., Estrada-Izquierdo, I. E. y Morales-Pérez, R. E. (2016). Logros de la educación ambiental y la sustentabilidad urbana en México. Revista Electrónica de Investigación Educativa, 18(3), 37-50; Morales Pérez, R. E. y Meraz Cabrera, L. (2015). Los mercados públicos en el Distrito Federal como espacio público, servicio urbano y generadores de alto volumen de residuos sólidos urbanos. En A. Ziccardi y A. González (coords.), Habitabilidad y política de vivienda en México (pp. 577-585). Ciudad de México: UNAM.

Fecha de recepción: 5 de septiembre de 2017.

Fecha de aceptación: 20 de diciembre de 2017. 
